Corrigendum

\title{
Corrigendum to "The Motivating Function of Healthcare Professional in eHealth and mHealth Interventions for Type 2 Diabetes Patients and the Mediating Role of Patient Engagement"
}

\author{
Guendalina Graffigna $\left(\mathbb{D},{ }^{1}\right.$ Serena Barello $\mathbb{D}^{1},{ }^{1}$ Andrea Bonanomi, ${ }^{2}$ and Julia Menichetti ${ }^{1}$ \\ ${ }^{1}$ Department of Psychology, Università Cattolica del Sacro Cuore, Largo A. Gemelli 1, 20123 Milan, Italy \\ ${ }^{2}$ Department of Statistical Sciences, Università Cattolica del Sacro Cuore, Largo A. Gemelli 1, 20123 Milan, Italy
}

Correspondence should be addressed to Guendalina Graffigna; guendalina.graffigna@unicatt.it

Received 30 July 2019; Accepted 21 August 2019; Published 20 November 2019

Copyright (c) 2019 Guendalina Graffigna et al. This is an open access article distributed under the Creative Commons Attribution License, which permits unrestricted use, distribution, and reproduction in any medium, provided the original work is properly cited.

In the article titled "The Motivating Function of Healthcare Professional in eHealth and mHealth Interventions for Type 2 Diabetes Patients and the Mediating Role of Patient Engagement" [1], the Morisky Medication Adherence Scales (MMAS-4) was wrongly mentioned. The correct scale used in this work is the Morisky Green Levine Scale (MGLS). The latter is an older, 4-item scale that is different from the MMAS-4, in particular in the formulation of the second item of the scale. Therefore, "Morisky Medication Adherence Scale" and "MMAS-4" should be corrected to "Morisky Green Levine Scale" and "MGLS" throughout the entire article.

\section{References}

[1] G. Graffigna, S. Barello, A. Bonanomi, and J. Menichetti, "The motivating function of healthcare professional in eHealth and mHealth interventions for type 2 diabetes patients and the mediating role of patient engagement," Journal of Diabetes Research, vol. 2016, Article ID 2974521, 10 pages, 2016. 


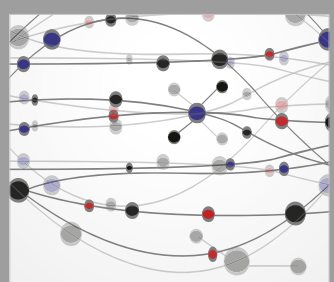

The Scientific World Journal
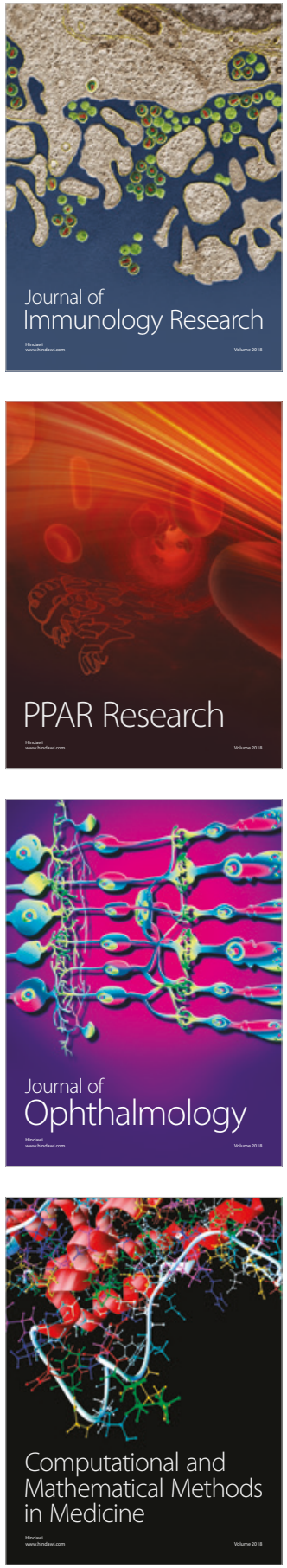

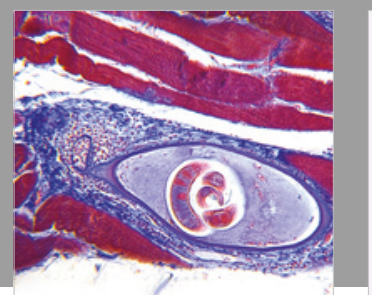

Gastroenterology Research and Practice

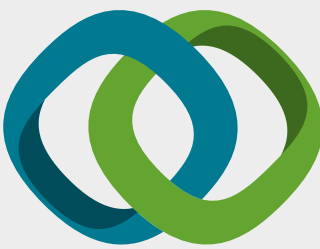

\section{Hindawi}

Submit your manuscripts at

www.hindawi.com
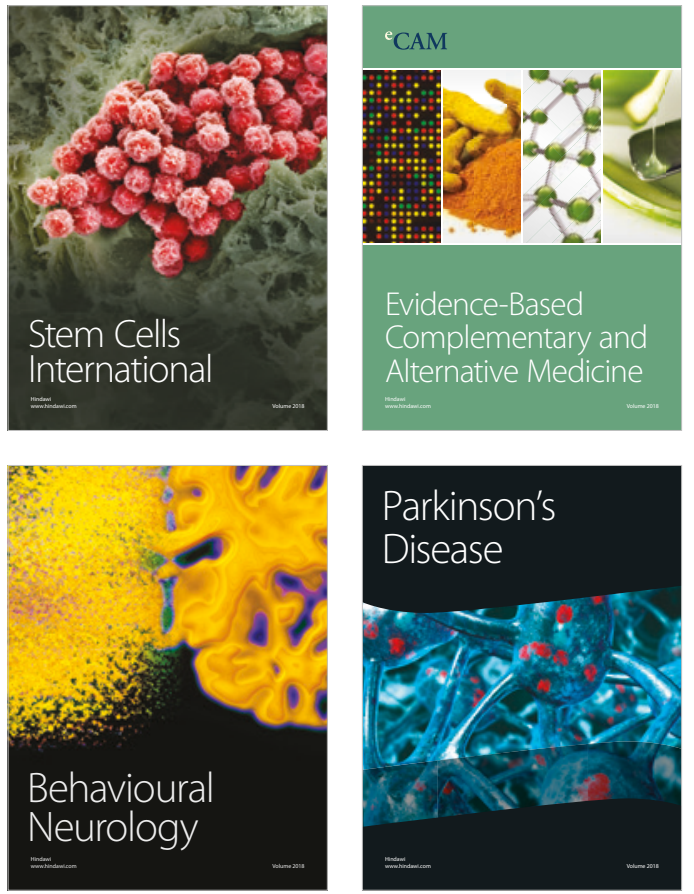

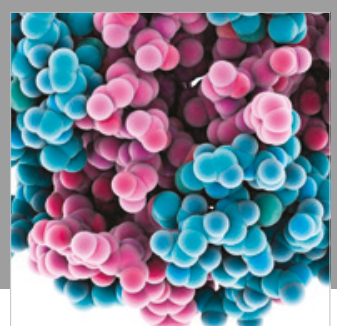

ournal of

Diabetes Research

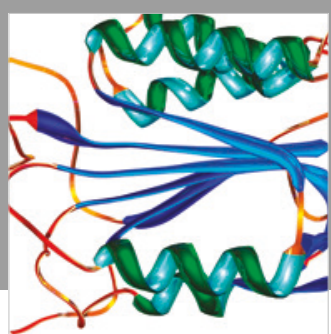

Disease Markers
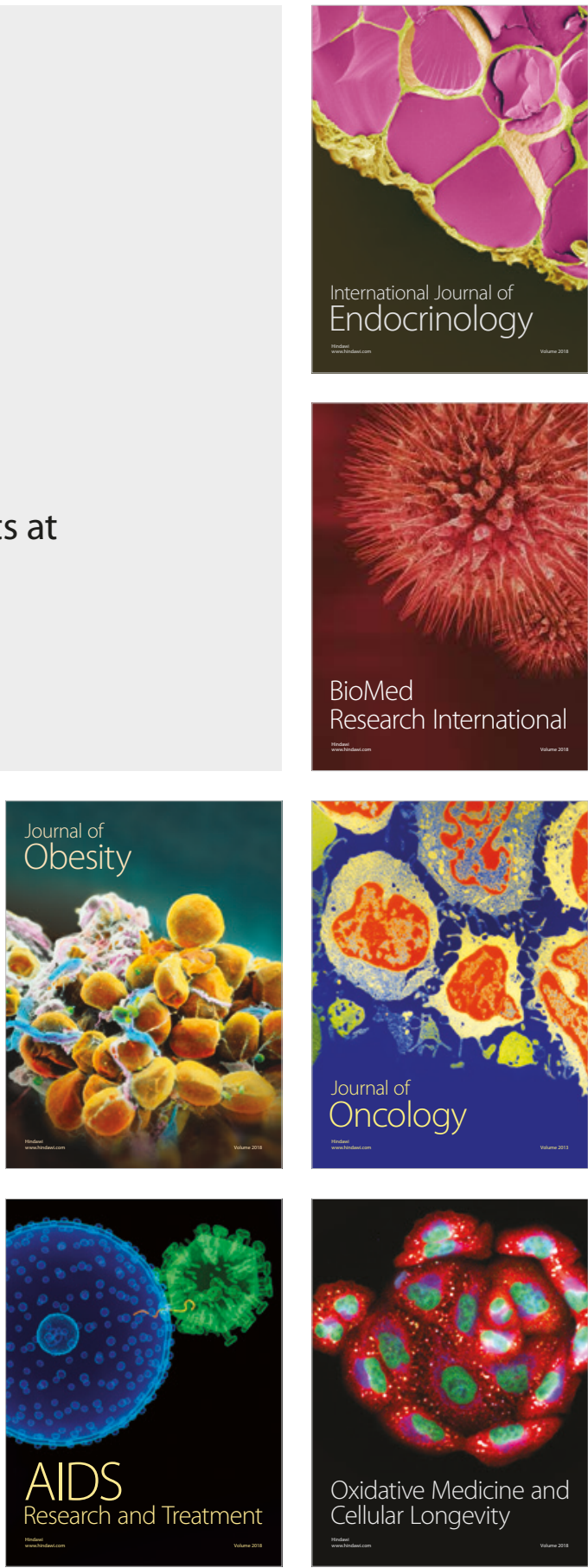